\title{
+SHELL MODEL CALCULATIONS AT SUPERDEFORMED SHAPES
}

W. Nazarewicz++

Joint Institute for Heavy Ion Research

Holifield Heavy Ion Research Facility

P.O. Box 2008, Oak Ridge, Tennessee 37831, USA

J. Dobaczewski

Institute of Theoretical Physics

Warsaw University, ul. Hoża 69, PL00681 Warsaw, Poland

P. Van Isacker

SERC, Daresbury Laboratory

Warrington WA4 4AD, United Kingdom

\begin{abstract}
+Invited Talk presented at the Workshop-Symposium on "Future Directions in Nuclear Physics with $4 \pi$ Gamma Detection Systems of the New Generation", 4-16 March 1991, Strasbourg, France

${ }^{++}$On leave of absence from Institute of Theoretical Physics, Warsaw University
\end{abstract}

Document no. 91-02

Institute Sponsors

The Triversity of Tennessee

Vanderbilt University

Oak Ridge National Laboratory
Institute Administration

The University of Tennessee 


\title{
SHELL MODEL CALCULATIONS AT SUPERDEFORMED SHAPES
}

\author{
W. Nazarewicz* \\ Joint Institute for Heavy-Ion Research, Holifield Heavy Ion Research Facility, \\ P.O. Box 2008, Oak Ridge, Tennessee 37831, U.S.A. \\ J. Dobaczewski \\ Institute of Theoretical Physics, Warsaw University, ul. Hoża 69, PL-00-681 \\ Warsaw, Poland \\ P. Van Isacker \\ SERC Daresbury Laboratory, Warrington WA4 4AD, U.K.
}

\begin{abstract}
Spectroscopy of superdeformed nuclear states opens up an exciting possibility to probe new properties of the nuclear mean field. In particular, the unusually deformed atomic nucleus can serve as a microscopic laboratory of quantummechanical symmetries of a three dimensional harmonic oscillator. The classifications and coupling schemes characteristic of weakly deformed systems are expected to be modified in the superdeformed world. The "superdeformed" symmetries lead to new quantum numbers and new effective interactions that can be employed in microscopic calculations. New classification schemes can be directly related to certain geometrical properties of the nuclear shape.
\end{abstract}

\section{INTRODUCTION}

The obsezvation of superdeformed states constitutes an important confirmation of the shell structure of the nucleus. This phenomenon is best understood in terms of the irregular distribution of the quantal energy levels in the nucleus forming "shells", i.e. bunches of c'ose-lying states. As is well known, the nuclear shell structure succesfully explaina most nuclear features such as the occurrence of especially stable systems (magic nuclei), the abundance of isotopes in nature, etc. What is, however, crucial in the search for the origin of superdeformation is the observation that pronounced shell structure may also appear in the extreme conditions of very large distortions. The quantum-mechanical shell correction, see e.g. refs. ${ }^{1,2}$, strongly fluctuating with particle number plays a very important role in determining the excitation energy of superdeformed structures.

Quantum-mechanically, the unusual stability of superdeformed states can be attributed to strong shell effects that are present in the average nuclear potential at the $2: 1$ shape. For example, in the harmonic oscillator model, the strongest level degeneracy occurs when the frequency ratio is a rational number and results in an appearance of spherical and superdeformed magic gaps and magic numbers. $2-4$

\footnotetext{
-On leave of absence from Institute of Theoretical Phyrica, Warwaw University
} 
Of course, the realistic single-particle spectrum is rather poorly reproduced by the spectrum of the harmonic oscillator. It looks rather "messy" with many levels crossing each other at different deformations. However, one can attempt to classify the single-particle states by introducing specific auxiliary quantum numbers. Firstly, one can observe that the single-particle orbitals can be divided into two groups, namely the "normal-parity" states strongly mixed by the deformed mean field and the "abnormal-parity" (unique-parity) intruder orbitals originating from the high- $j$, high- $N$ subshells. These subshells are moved down in energy partly due to the spin-orbit interaction and partly due to the flat bottom of the average field. Because of their opposite parity the intruder states do not mix with the normal-parity orbitals and therefore can be approximately interpreted as pure- $j$ shell model states.

The superdeformed bands observed so far present significant variations both with particle number and angular momentum. The calculations based on the deformed shell model theory ${ }^{5-7}$ explain many of the observed properties of superdeformed bands in terms of intruder orbitals originating from the high- $N$ oscillator shells and approaching the Fermi surface at large deformations. These states, i.e. $N=7$ neutrons and $N=6$ protons, carry large intrinsic quadrupole moments and therefore they lead to significant shape-polarisation effects causing deformation variations between different super' : large intrinsic angular momentum they are strongly influenced by the Coriolis force. The Nilsson model cranking calculations by Bengtsson et al. ${ }^{7}$ were able to attribute the observed variations in the dynamical moments of inertia in the superdeformed bands to the number of high- $N$ intruder states occupied. The different high- $N$ occupations have also bec:. shown to explain deformation variations with the particle number. Therefore, high-spin spectroscopy of superdeformed bands provides us with a valuable information about the intruder content of superdeformed intrinsic states.

Interestingly enough, the combined effect of the spin-orbit interaction and the flat bottom of the average field (or the $\ell^{2}$-term in the Nilsson model) leads to a very small energy splitting between certain spherical normal-parity subshells. For instance, it has been observed that certain pairs of normal-parity states are very close to each other and have very similar deformation dependence. These orbitals originating from the normal parity subshells with $j=\ell-\frac{1}{2}$ and $j^{\prime}=(\ell-2)+\frac{1}{2}$ can thus be viewed as pseudo spin-orbit doublets with $j=i \pm \frac{1}{2}$ and identical pseudo-orbital anguiar momentum $\ell_{.} 8,9$

In an attempt to find a powerful coupling scheme for shell-model-like calculations one can go even further and ignore the spherical splitting between naturalparity states. (Because of the large energy splitting between natural-parity states and the unique-parity high- $j$ intruder subsheli with $j=N$, the latter orbitals do not belong to this coupling scheme.) This symmetiy, referred to as pseudo-SU(3), proved to be very powerful in microscopic calculations. Indeed it has been demonstrated, see e.g. ref. ${ }^{10}$, that natural-parity orbitals can indeed be reasonably 
classified using the leading pseudo $\mathrm{SU}(3)$ representations. 11,12

In the pseudo SU(3) limit the natural parity Nilsson orbitals form a psentooscillator spectrum which can be labelled using the pseudo-8symptotic quantum numbers $\tilde{N}=N-1, \bar{n}_{z}=n_{z}$. Moreover, the pairs of Nilsson levels $\left[N n_{z} \Lambda\right] \Omega=\Lambda+\frac{1}{2}$, $\left[N n_{z} \Lambda+2\right] \Omega=\Lambda+\frac{3}{2}$ can be viewed as pseudo spin-orbit doublets $\left[\bar{N}_{n_{z}} \bar{\Lambda}\right] \Omega=\bar{\Lambda} \pm \frac{1}{2}$ with $\bar{\Lambda}=\Lambda+1$. Such pairs of orbitals are usually very close to each other due to the very small pseudo spin-orbit splitting, and they have very similar particle number dependence and deformation behaviour. Experimentally, pseudo spinorbit doublets show up clearly in the systematics of single-particle excitations. 13

At the large deformations characterizing superdeformed configurations the pseudo $S U(3)$ scheme is still expected to be valid since the high- $j$ intruder orbitals seem to be well separated from the natural parity states, see also refs. ${ }^{11}, 14$ Since the normal-parity orbitals form a pseudo-oscillator pattern, they form regions of high level density at 2:1 shapes. The unique-parity intruder orbitals cross the pseudo-oscillator shell closures forming the actual superdeformed gaps seen in realistic single-particle spectra. This simple mechanism explains in a natural way the abundance and sistematics of large single-particle gaps at superdeformed shapes 15 as well as the presence of "twinned" bands in the $A \approx 150$ mass region. 16

Since the normal-parity states form a pseudo-oscillator pattern, one can argue that many properties of superdeformed states should reflect basic features of the harmonic oscillator. In section 2 we shall discuss some properties of the singleparticle shell structure of a three-dimensional harmonic oscillator with frequencies in rational ratios ( $\mathrm{RHO}$ ).

The problem of unusual degeneracies of the single-particle levels in the RHO has been extensively discussed in the literature. Clasically, the symmetry group of the RHO has been first discussed already in 1940 by Jauch and Hill 17 who demonstrated that for the 2-dimensional anisotropic RHO the underlying symmetry group was isomorphic to $\mathrm{SU}(2)$. Twenty five years later Dulock and McIntosh 18 made a very careful investigation of this case. They found $\mathrm{SU}(2)$ to be the symmetry group of the RHO and discussed the corresponding canonical transformation and the constants of the motion.

The degeneracy of the quantal $\mathrm{N}$-dimensional isotropic harmonic oscillator was discussed by Demkov in $195419^{19}$ and $1959^{20}$ and by Baker in $1956^{21}$ who explained it in terms of the $\mathrm{N}$-dimensional unitary group. The symmetry group of the two-dimensional quantum-mechanical $\mathrm{RHO}, \mathrm{SU}(2)$, has been worked out by Demkov in $1963^{22}$. As an example he discussed the case of the RHO with the 2:1 frequency ratio. In the conclusions of his paper, however, he stated explicitly that for the $\mathrm{N}$-dimer sional RHO the minimal symmetry group would be $\mathrm{SU}(\mathrm{N})$. The general case of the $\mathrm{RHO}$ in $\mathrm{N}$-dimensions was investigated by Maiella in 1967 23 in the classical picture and then by Vendramin in 196824 and Maiella and Vilasi in 196925 , in the quantum mechanical picture. They derived the canonical transformation and introduced the generalized Bose operators that could reduce (or increase) the number of oscillator quanta by $k$. Duimio and Zambotti in 1966 
26 and Brandt and Greenberg in $1969{ }^{27}$ studied generalized Bose operators and found their power-series representations. They consider the finite- $k$ values, but also generalized the formalism to the case of $k \rightarrow \infty$. (More discussion concerning the two-dimensional RHO can be found in works by Il'kaeva ${ }^{28}$, Cisneros and McIntosh 29 and Louck, Moshinky and Wolf ${ }^{30}$. There is also a beautiful review paper on symmetry and degeneracy by McIntosh 31 where additional references can be found).

Finally, the connection between the symmetry algebra of the RHO and nuclear superdeformation has been pointed out by Rosensteel and Draayer in $1989^{32}$ (see also ref. ${ }^{33}$ ).

\section{SHELL STRUCTURE OF THE DEFORMED HARMONIC OSCILLATOR}

The single-partic'e Hamiltonian of the three-dimensional oscillator potential is given by

$$
\hat{H}=\frac{1}{2} \sum_{i=1}^{3}\left(p_{i}^{2}+\omega_{i}^{2} x_{i}^{2}\right)=\frac{1}{2} \sum_{i=1}^{3} \omega_{i}\left\{a_{i}, a_{i}^{+}\right\},
$$

where we assume $M=1, \hbar=1$.

The single-particle energies of (1) are

$$
e_{n_{1}, n_{2}, n_{3}}=\sum_{i=1}^{3} \omega_{i}\left(n_{i}+\frac{1}{2}\right)
$$

where $n_{i}$ are numbers of oscillator quanta in three spatial directions and the corresponding Hilbert space, $\mathcal{H}$, is spanned by the kets

$$
|\hat{n}\rangle=\left|n_{1} n_{2} n_{3}\right\rangle=\frac{1}{\sqrt{n_{1} ! n_{2} ! n_{3} !}}\left(a_{1}^{+}\right)^{n_{1}}\left(a_{2}^{+}\right)^{n_{2}}\left(a_{3}^{+}\right)^{n_{3}}|-\rangle .
$$

The single-particle diagram of the axially-deformed harmonic oscillator is displayed in fig. 1. Here $\omega_{1}=\omega_{2}=\omega_{\perp}$ and the single-particle energies depend only on two quantum numbers: $n_{1}=n_{1}+n_{2}$ and $n_{3}$. Due to the axial symmetry each level is $\left(n_{\perp}+1\right)$ times degenerate. By introducing the deformation parameter $\epsilon=\left(\omega_{\perp}-\omega_{3}\right) / \omega_{0}$ eq. (2) can be written as

$$
e_{n_{1}, n_{3}}=\omega_{0}\left[N+\frac{3}{2}-\frac{1}{3} \varepsilon\left(2 n_{3}-n_{1}\right)\right] .
$$

Here $N=n_{\perp}+n_{3}$ is the total number of oscillator quanta (the principal oscillator quantum number) and $\omega_{0}=\frac{1}{3}\left(2 \omega_{\perp}+\omega_{3}\right)$. It is immediately seen from eq. (2) that orbicals with the same value of $\omega_{1} n_{1}+\omega_{3} n_{3}$ are degenerate.

For the RHO the ratios of frequencies are rational numbers. This condition can be expressed in terms of three integers $k_{i}, i=1,2,3$ :

$$
\omega_{i} k_{i}=\bar{\omega},
$$




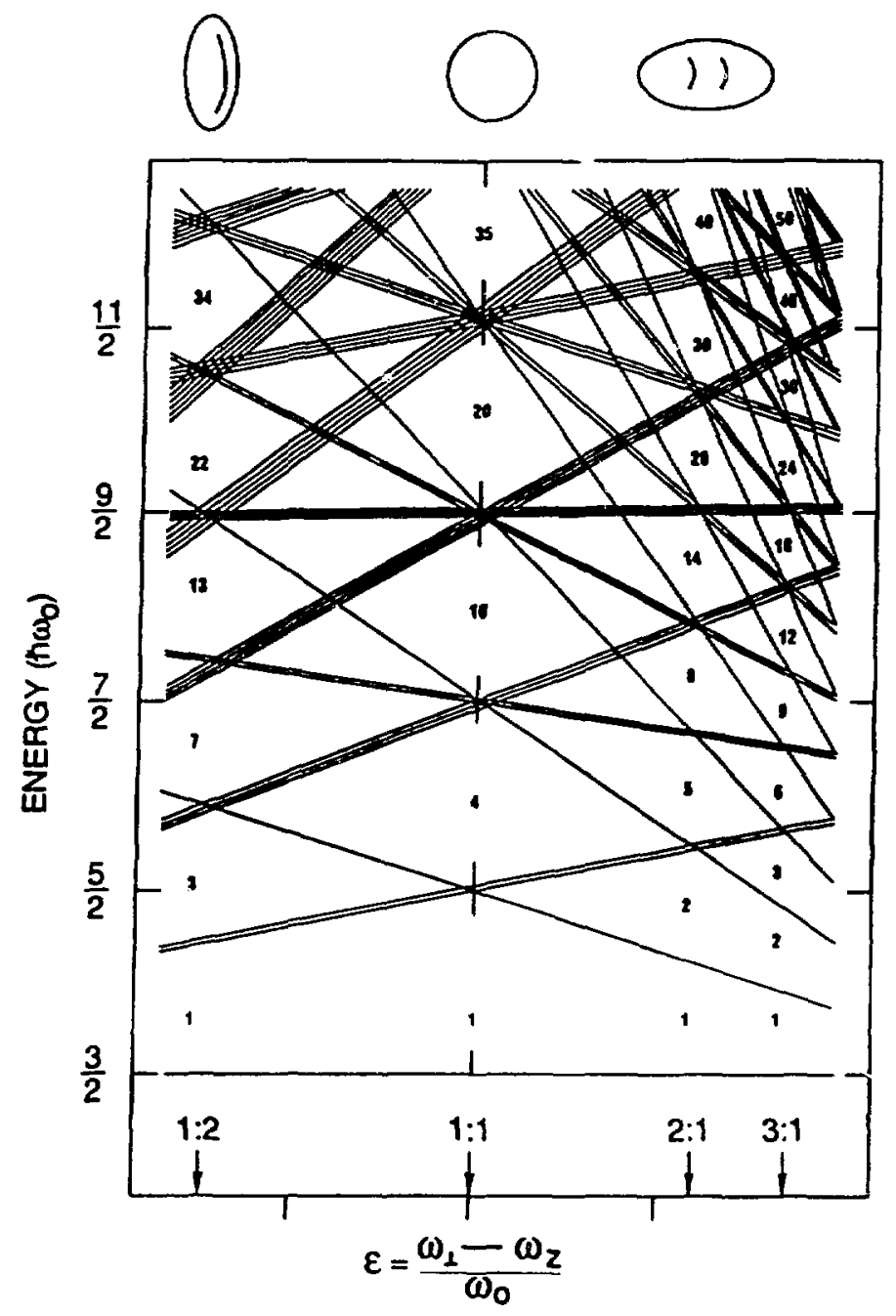

Fig. 1. Single-particle level spectrum of the axially symmetric harmonic oscillator shown as a function of quadrupole deformation $\varepsilon$. The orbital degeneracy is $n_{\perp}+1$, which is illustrated by artificially splitting the lines. The arrows indicate the characteristic deformations corresponding to the ratio of $\omega_{\perp}: \omega_{3}=1: 2,1: 1,2: 1$ and 3:1.

where $\bar{\omega}$ can be calculated from the volume conservation condition, $\omega_{1} \omega_{2} \omega_{3}=\bar{\omega}_{0}^{3}$, and is equal to

$$
\bar{\omega}=\sqrt[3]{k_{1} k_{2} k_{3}} \cdot \dot{\omega}_{0}, \quad \bar{\omega}_{0}=\frac{41}{A^{1 / 3}}
$$

For example, the spherical shape corresponds to $k_{1}=k_{2}=k_{3}$, while the axial shapes have $k_{1}=k_{2}=k_{1}$. In the axial case it is convenient to write the singleparticle energies in terms of the shell frequency, $w_{\text {shell, }}$ and the shell principal 
quantum, $N_{\text {shell }}$, defined by ${ }^{2}$ :

$$
\omega_{\perp} n_{\perp}+\omega_{3} n_{2}=\omega_{\text {shell }} N_{\text {shell }}, N_{\text {shell }}=n_{1} k_{3}+n_{3} k_{\perp}, \omega_{\text {shell }}=\frac{\tilde{\omega}}{k_{\perp} k_{3}} .
$$

Let us now introduce new quantum numbers $\nu_{i}$ and $\lambda_{i}$ :

$$
n_{i}=k_{i} \nu_{i}+\lambda_{i}, \quad \nu_{i}=\left[\frac{n_{i}}{k_{i}}\right], \quad \lambda_{1}=n_{i}\left(\bmod k_{i}\right),
$$

where the symbol $[x]$ stands for the integer part of $x$. Single-particle energies of the RHO can be thus written as

$$
e_{\Lambda_{i} \lambda_{1} \lambda_{2} \lambda_{3}}=\bar{\omega} M+\tilde{\omega} \sum_{i=1}^{3} \frac{\lambda_{i}+\frac{1}{2}}{k_{i}}
$$

where $M=\nu_{1}+\nu_{2}+\nu_{3}$ is the new principal quantum number. At fixed values of $\lambda_{i}$ the level degeneracy is equal to $\frac{1}{2}(M+1)(M+2)$, i.e. it corresponds exactly to the degeneracy of spherical oscillator with principal quantum number $M$. This fact suggests that the "hidden" symmetry of the quantum-mechanical RHO should be exactly the same as the dynamical symmetry of the isotropic harmonic oscillator, namely $\mathrm{SU}(3)$. In order to show this explicitely one can introduce new ladder operators $27 \dagger$

$$
A_{i}^{\{\lambda\}}=\frac{1}{\sqrt{k_{i}}}\left(a_{i}\right)^{k_{i}}\left(\frac{\hat{n}_{i}-\lambda_{1}}{\hat{n}_{i}\left(\hat{n}_{i}-1\right) \ldots\left(\hat{n}_{i}-k_{i}+1\right)}\right)^{\frac{1}{2}}=\left(a_{i}\right)^{k_{i}}\left(\left[\frac{\hat{n}_{i}}{k_{i}}\right] \frac{\left(\hat{n}_{i}-k_{i}\right) !}{\hat{n}_{i} !}\right)^{\frac{1}{2}}
$$

or, alternatively 26.24

$$
A_{i}^{\{\lambda\}}=\frac{1}{\sqrt{k_{i}}}\left(\frac{1}{\sqrt{a_{i} a_{i}^{+}}} a_{i}\right)^{k_{i}-\lambda_{i}-1} a_{i}\left(\frac{1}{\sqrt{a_{i} a_{i}^{+}}} a_{i}\right)^{\lambda_{i}}
$$

where $\hat{n}_{1}=a_{i}^{+} a$, is the boson number operator and $\{\lambda\} \equiv\left\{\lambda_{1} \lambda_{2} \lambda_{3}\right\}$. It is easy to verify that operators (10) indeed fulfil the standard boson commutation rules, i.e.

$$
\left[A_{i}^{\{\lambda\}}, A_{j}^{(\lambda\}+]}=\delta_{i, j} .\right.
$$

The new boson operator $A_{i}^{(\lambda)}$ acts only on the quantum number $\nu_{i}$ leaving $\lambda_{i}$ unchanged,

$$
A_{j}^{(\lambda)+}\left|\cdots, \nu_{i} k_{i}+\lambda_{i}, \cdots\right\rangle=\sqrt{\nu_{i}+1}\left|\cdots,\left(\nu_{i}+1\right) k_{i}+\lambda_{i}, \cdots\right\rangle .
$$

'These operators have been employed by Rasetti ${ }^{34}$ to generate fields of anharmonicities for the quantum-mechanical harmonic oscillator. In atomic physic the operators (10) are referred to as many-phonon generalied Bose operators 35, 36. They appear in the context of multiphoton generalised Holstein-Primakoff squeesed atakes. 
This means that the Hilbert space of the RHO can be decomposed as

$$
\mathcal{H}=\sum_{\{\lambda\}} \mathcal{H}^{[\lambda]}
$$

where the subspace $\mathcal{H}^{(\lambda)}$ is spanned by kets (3) with a fixed value of $\{\lambda\}$. In this new subspace the RHO Hamiltonian becomes

$$
H=\frac{1}{2} \tilde{\omega} \sum_{i=1}^{3}\left\{A_{i}^{[\lambda]}, A_{i}^{[\lambda]+}\right\}-\frac{1}{2} \sum_{i=1}^{J} \omega_{i}\left(k_{1}-2 \lambda_{i}-1\right),
$$

i.e. formally looks like the Hamiltonian of the isotropic harmonic oscillator (up to an additive constant). Now, analogously to the spherical case, one can construct eight generators linear in $\left\{A_{i}^{\{\lambda\}}, A_{i}^{[\lambda]+}\right\}$ that fulfil the commutation rules of $\mathrm{SU}(3)$ and commute with the Hamiltonian (15). This completes the proof that SU(3) is indeed the dynamical symmetry in question. The RHO eigenstates belonging to the same $\{\lambda\}$ family form for a given $M$ the basis of an irreducible symmetric representation (irrep) of $\mathrm{SU}(3),(M,\{\lambda\})^{\ddagger}$. Each family has a corresponding ground state belonging to the one-dimensional representation of $\mathrm{SU}(3)$ for $M=0$, which is the vacuum for the new bosons (10). Since $0 \leq \lambda_{i}<k_{i}$ the number of $\{\lambda\}$-families is equal to $k_{1} k_{2} k_{3}$ and becomes large when the frequency ratios are not very commensurable $e^{\S}$.

Fig. 2 shows a typical example of the RHO spectrum in the case of $k_{1}=1$, $k_{2}=2, k_{3}=3$. The levels can be grouped into six independent $\{\lambda\}$-families, each forming the isotropic oscillator spectrum with frequency $\bar{\omega}$.

At the spherical shape $k_{1}=k_{2}=k_{3}=1$ there is only one family present, labelled by $\{\lambda\}=(000)$. The degeneracy of each level is $\frac{1}{2}(M+1)(M+2)$ and the magic gaps occur at particle numbers

$$
N_{\Lambda I}=\frac{1}{6}(M+1)(M+2)(M+3)=1,4,10,20,35,56,84, \ldots
$$

Other cases of significant physical interest are the superdeformed prolate $\left(k_{1}=1, k_{2}=1, k_{3}=2\right)$ and the hyperdeformed prolate $\left(k_{1}=1, k_{2}=1, k_{3}=3\right)$ shapes. Here, since $\lambda_{1}=\lambda_{2}=0$, the number of independent SU(3) irreps for given $M$ is simply equal to $k_{3}$ and they can be easily distinguished by means of $\lambda_{3}$.

As seen in fig. 3 there are two kinds of closed-shell systems that are expected at superdeformed shapes. In the "asymmetric" case, indicated as $A$, the number of filled shells within the family $(\lambda)=(000)$ is larger by one than that within the family $(\lambda)=(001)$. In the fig. 3 this is illustrated by the position of the Fermi level $\epsilon_{F}^{4}$. Consequently the magic numbers are then equal to sums of two consecutive spherical magic numbers and read $N_{\Lambda I+1}^{(000)}+N_{\Lambda I}^{(001)}=1,5,14,30,55$, etc. The numbers shown in the figure have been doubled to include the spin degeneracy. In the

\footnotetext{
I In the following the second SU(3) quantum number $\mu=0$ will be omitted

For more discussion concerning the transition to the case of incommensurable frequency ratio, we refer the reader to Cisneros and MeIntosh, ref. 29
} 
TRIAXIAL HARMONIC OSCILLATOR

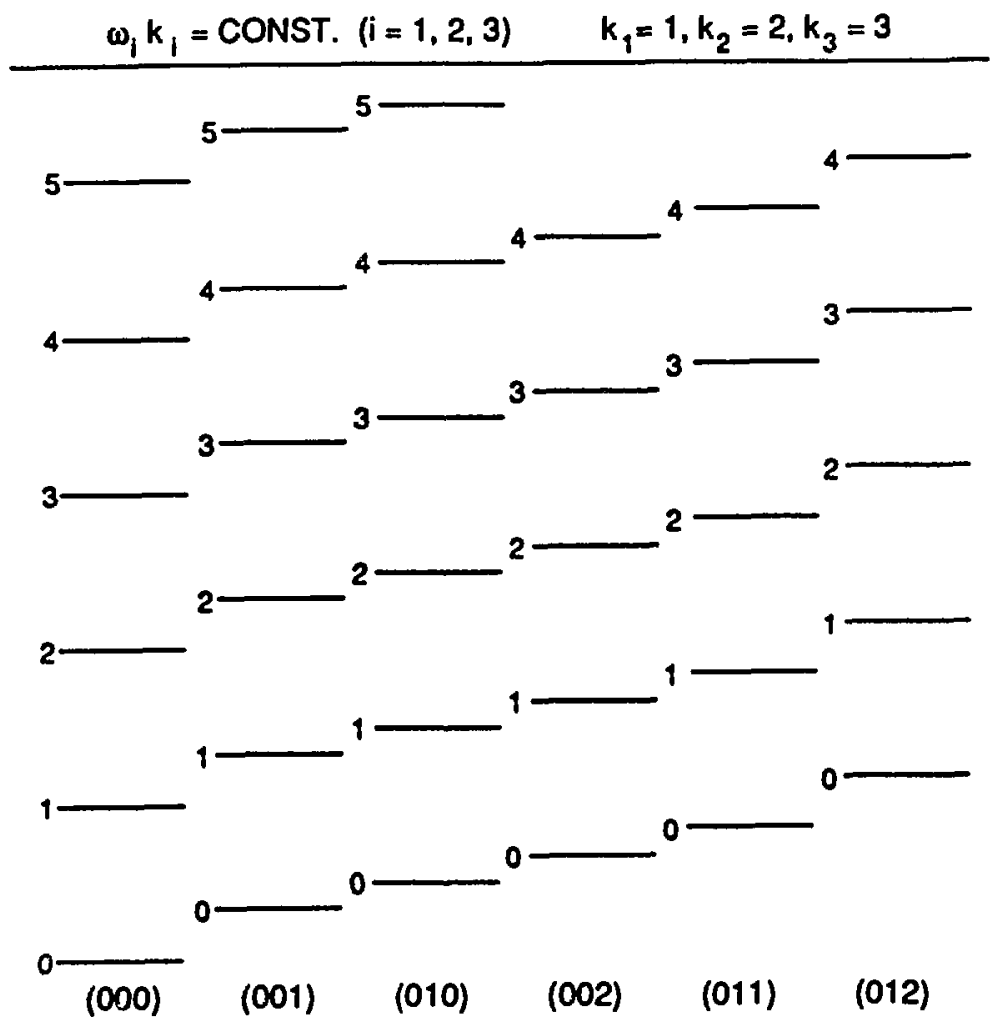

Fig. 2. Spectrum of the RHO with $k_{1}=1, k_{2}=2, k_{3}=3$. Each level is labelled by means of the principal quantum number $M$ and quantum numbers $\left(\lambda_{1} \lambda_{2} \lambda_{3}\right)$ that label different irreducible representations of SU(3). Except the usual SU(3) degeneracy, $\frac{1}{2}(M+1)(M+2)$, no additional degeneracies are present.

"symmetric" variant $B$ the missing (001) shell is filled and the magic numberz are equal to doubled spherical oscillator magic numbers, $N_{M}^{(000)}+N_{M I}^{(001)}=2,8,20,40$, 70, etc.

The situation becomes slightly more complex at hyperdeformed shapes, see fig. 4. In the "strongly asymmetric" variant $A$ the number of filled shells within the family (000) is larger by one than those of the families (001) and (002), which lesds to magic numbers $N_{M+1}^{000}+N_{M}^{001}+N_{M I}^{002}=1,6,18,40,75$, etc. In the varient $B$ the occupation of the family (002) is lower than those of the families (000) and (001) and the resulting magic numbers are $N_{M+1}^{000}+N_{M+1}^{001}+N_{M f}^{002}=2, S, 24,50$, 90, etc. Finally, in the "symmetric" case $C$ the occupations of families (000), (001) and (002) are identical and the magic numbers are equal to triplsd spherical oscillator magic numbers, $N_{N I}^{000}+N_{A}^{001}+N_{M}^{002}=3,12,30,60$ and 105, etc.

The examples discussed so far represent the simplest possible situation in 


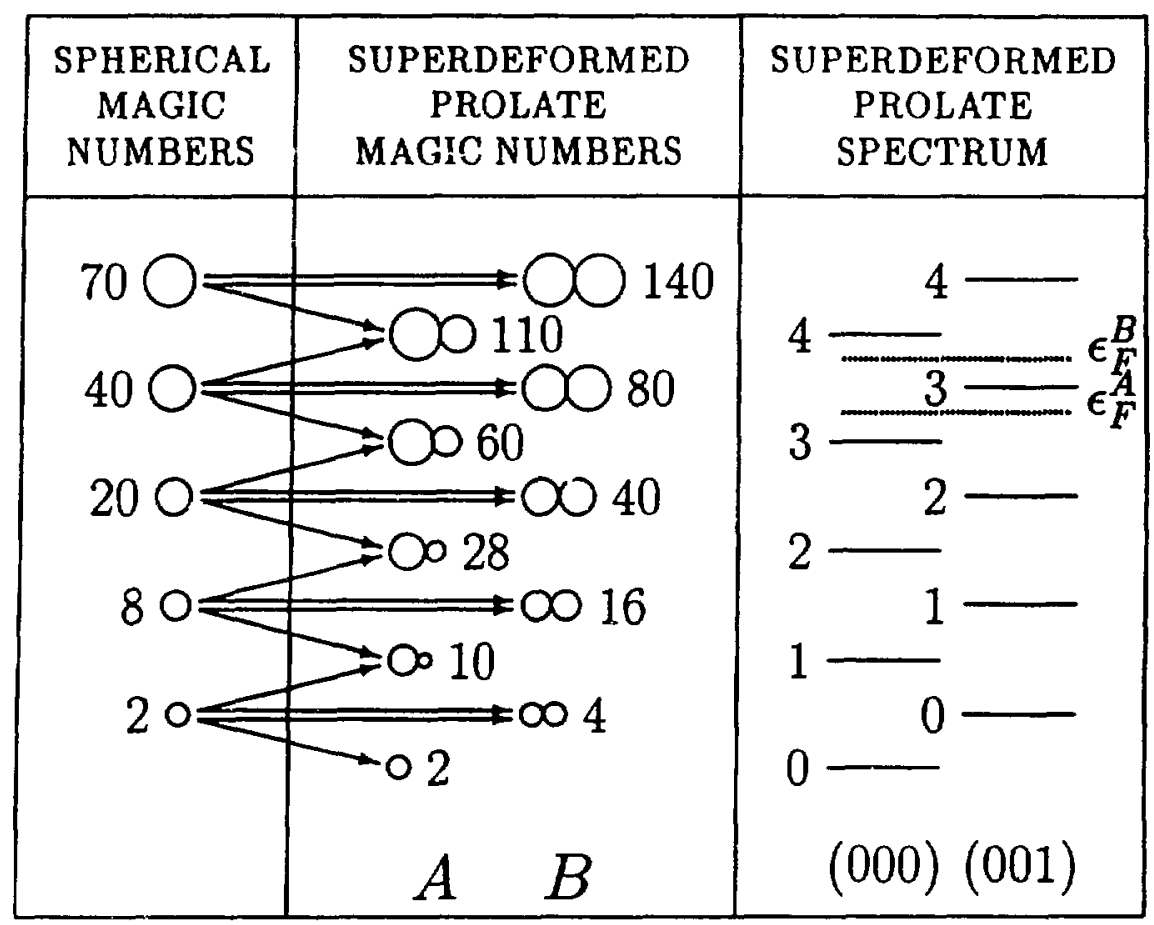

Fig. 3. Magic numbers and spectrum of the prolate superdeformed $\left(k_{1}=1, k_{2}=1\right.$, $k_{3}=2$ ) harmonic oscillator. The arrows indicate from which spherical oscillator representations a given superdeformed representation is built (see text). The sizes of circles schematically illustrate the dimensions of spherical representations. The harmonic oscillator degeneracies have been doubled to take into accont the spin. Two different positions of the Fermi level, $\epsilon_{F}^{4}$ and $\epsilon_{F}^{B}$, are indicated in the spectrum. The quantum numbers $\left(\lambda_{1}, \lambda_{2}, \lambda_{3}\right)$ of two $\lambda$-families are shown below the spectrum.

which the numbers $k_{i}$ are relatively prime. The degeneracy pattern becomes, hnvewer, rather complicated in the case when two $k_{i}$ 's have common multiplier. Let us consider, for instance, the case of oblate superdeformed shapes with $k_{1}=k_{2}=k_{1}>$ $1, k_{3}=1$. The number of one-dimensional irreps of SU(3) is equal to $k_{\perp}^{2}\left(0 \leq \lambda_{1}<\right.$ $\left.k_{1}, 0 \leq \lambda_{2}<k_{2}, \lambda_{3}=0\right)$ and the eigenstates (9) are given by:

$$
e_{M, \lambda_{1} \lambda_{2} 0}=\dot{\omega}\left(M+\frac{1}{2}\right)+\dot{\omega}\left(\frac{\lambda_{1}+\lambda_{2}+1}{k_{\perp}}\right) .
$$

It is seen that due to the equality of energies

$$
e_{A I_{1} \lambda_{1} \lambda_{2} \mathrm{O}}=e_{A I} I_{i} \lambda_{1}^{\prime} \lambda_{2}^{\prime} 0,
$$




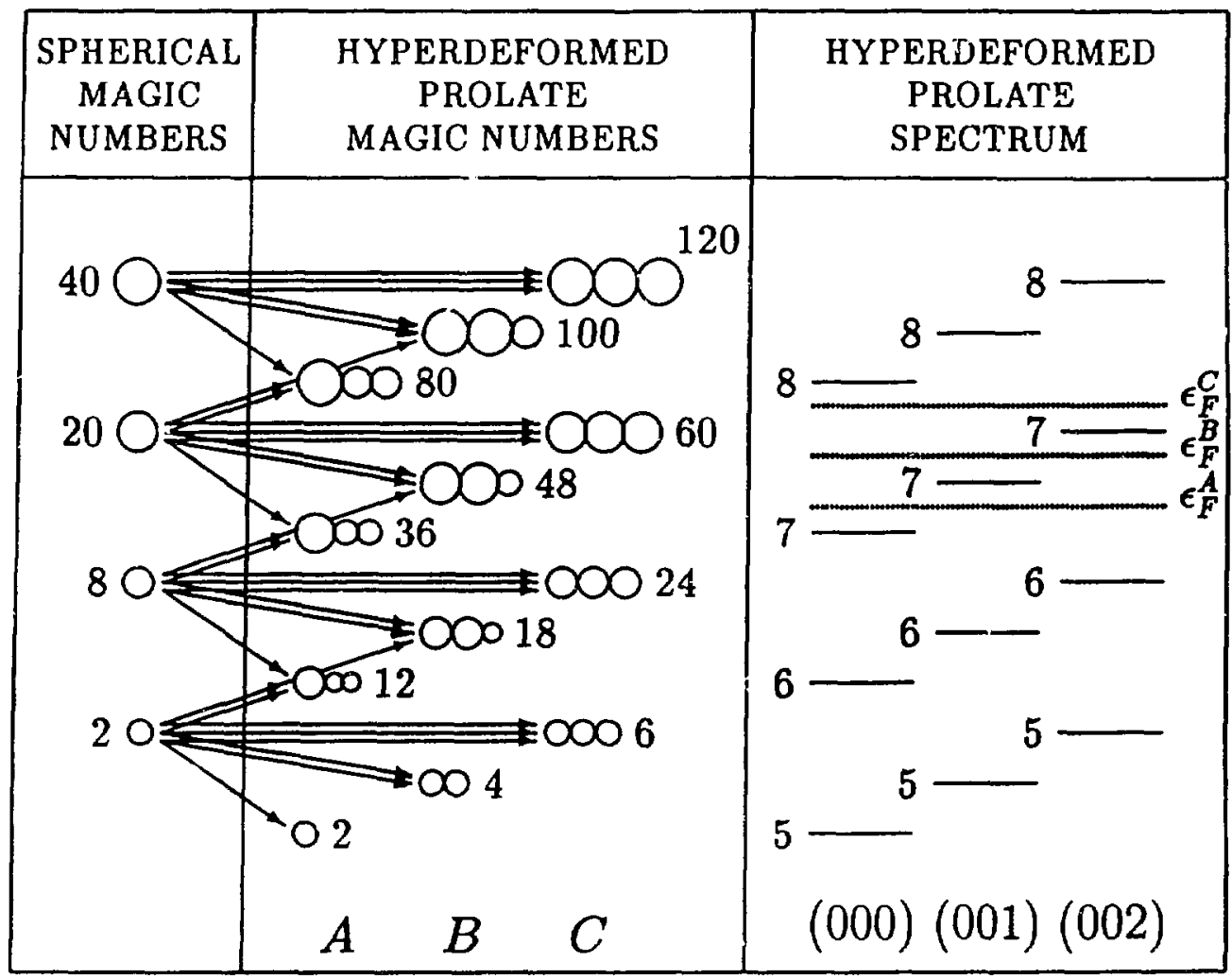

Fig. 4. Similar to fig. 4 but for the prolate hyperdeformed $\left(k_{1}=1, k_{2}=1, k_{3}=3\right)$ harmonic oscillator. Three different positions of the Fermi level, $\epsilon_{F}^{A}, \epsilon_{F}^{B}$, and $\epsilon_{F}^{c}$, are indicated in the spectrum. The quantum numbers $\left(\lambda_{1} \lambda_{2} \lambda_{3}\right)$ of three $\lambda$-families are shown below the spectrum.

which occur for

$$
M^{\prime}=M+\left[\frac{\lambda_{1}+\lambda_{2}}{k_{\perp}}\right] \text { and } \lambda_{1}^{\prime}+\lambda_{2}^{\prime}=\lambda_{1}+\lambda_{2}\left(\bmod k_{\perp}\right),
$$

additional degeneracies, not accounted for by the dynamical SU(3) symmetry, are present. Fig. 5 shows the single-particle spectrum of the RHO for $k_{\perp}=2$. According to eq. (18) the levels belonging to pairs of irreps $(M, 010)-(M, 100)$ and $(M+1,000)-(M, 110)$ are degenerate. Since the degeneracy of the energy levels (in the discussed case: $1,2,4,6,9, \cdots$ ) does not coincide with the dimension of any irrep of SU(3) it led some authors, see e.g. ${ }^{24}$, to the conclusion that $\mathrm{SU}(3)$ is not the dynamical symmetry of the RHO, but some more general symmetry has to be present that would explain observed degeneracies. This suggestion has 
been questioned by Maiella and Vilasi 25 who demonstrated that, except for particular cases, each energy level of the RHO corresponds to a reducible (rrep) representation of $S U(3)$.

\section{SUPERDEFORMED OBLATE}

$$
k_{1}=2, k_{2}=2, k_{3}=1
$$

\begin{tabular}{|c|c|c|c|c|}
\hline$N_{\text {SHELL }}$ & DEGEN. & $\begin{array}{l}\text { IRREP SU(3) } \\
\left(M_{1} \lambda_{1} \lambda_{2} \lambda_{3}\right) \\
\end{array}$ & $\begin{array}{c}\operatorname{RREP} \operatorname{SU}(3) \\
\left(\widetilde{N}, \lambda_{\perp}\right) \\
\end{array}$ & $\begin{array}{c}\text { IRREP O(4) } \\
\left(j^{+}, j^{-}\right) \\
\end{array}$ \\
\hline 4 & 9 & $(1,110),(2,000)$ & $(2,0)$ & $(1,1)$ \\
\hline 3 & 6 & $(1,010),(1,100)$ & $(1,1)$ & $\left(1, \frac{1}{2}\right)$ \\
\hline 2 & 4 & $(0,110),(1,000)$ & $(1,0)$ & $\left(\frac{1}{2}, \frac{1}{2}\right)$ \\
\hline 1 & 2 & $(0,100),(0,010)$ & $(0,1)$ & $\left(\frac{1}{2}, 0\right)$ \\
\hline 0 & 1 & $(0,000)$ & $(0,0)$ & $(0,0)$ \\
\hline
\end{tabular}

Fig. 5. Spectrum of the RHO with $k_{1}=k_{2}=2, k_{3}=1$ (superdeformed oblate shape). The principal shell quantum number $N_{\text {shell }}$ is defined by means of eq. (7). Each level is labelled by means of the irrep SU(3) labels $\left(M, \lambda_{1} \lambda_{2} \lambda_{3}\right)$, the rrep SU(3) labels $\left(\tilde{N}, \lambda_{\perp}\right)$, and the irrep $O(4)$ labels $\left(j^{+}, j^{-}\right)$, see text.

In order to label the rreps of SU(3), one can introduce quantum numbers $\lambda_{\perp}$ and $\bar{N}$ defined in a similar way as $\lambda_{i}$ (see e.g. ${ }^{37}$ ):

$$
\lambda_{\perp}=\nu_{\perp}\left(\bmod k_{\perp}\right), \quad \bar{N}=\nu_{\perp}+\nu_{3},
$$

where $\nu_{\perp}=\left[n_{\perp} / k_{\perp}\right]$. Contrary to the ease of prolate shapes the shell degeneracy depends explicitly on $\lambda_{\perp}$ :

$$
n\left(\bar{N}, \lambda_{\perp}\right)=\frac{1}{2}(\bar{N}+1)\left[k_{\perp} \tilde{N}+2\left(\lambda_{\perp}+1\right)\right]
$$

(for the general case of $k_{3} \neq 1$ see ref. ${ }^{24}$ ). The total number of states in an oblate superdeformed magic system (counting from the bottorn and including the last 
$\left(\bar{N}, \lambda_{\perp}\right)$ shell) is then equal

$$
\begin{gathered}
N_{\dot{N}, \lambda_{\perp}}=\frac{1}{2}\left(\lambda_{\perp}+1\right)\left(\lambda_{\perp}+2\right) N_{\hat{N}}+\left[\frac{1}{2} k_{\perp}\left(k_{\perp}-1\right)+\left(\lambda_{\perp}+2\right)\left(l-\lambda_{\perp}-1\right)\right] N_{\dot{N}-1} \\
+\frac{1}{2}\left(k_{\perp}-\lambda_{\perp}-1\right)\left(l-\lambda_{\perp}-2\right) N_{\dot{\Lambda}-2},
\end{gathered}
$$

where $N_{\hat{\Lambda}}$ is given by eq. (16).

For $k_{1}=2$ the third term in eq. (22) vanishes and the magic numbers can be thus divided into two groups with $\lambda_{\perp}=0$ or 1

$$
N_{\dot{N}, \lambda_{\perp}}= \begin{cases}N_{\dot{N}}+3 N_{\dot{N}-1}=1,7,22,50, \cdots & \text { if } \lambda_{\perp}=0 \\ 3 N_{\dot{N}}+N_{\dot{N}-1}=3,13,34, \cdots & \text { if } \lambda_{\perp}=1\end{cases}
$$

Unfortunately, the answer in terms of the rreps of SU(3) is not very appealing. As nicely expressed by Mclntosh ${ }^{31}$, One could never be sure that even in those systems for which the degeneracy was supposedly resolved, the day would never come when an even bigger group would be discovered, producing the same pattern of accidental degeneracies, but encompassing constants of the motion which could be important in some as yet unforeseen context. Interestingly, such a group has been found in the discussed case of oblate superdeformed shapes.

In 1967 Ravenhall, Sharp and Pardee ${ }^{38}$ demonstrated that the imposition of a reflection condition can change $S U(3)$ to $O(4)$, and vice versa. In particular, they analysed the isotropic harmonic oscillator with an impenetrable barrier across the symmetry (say $x y$ ) plane. The eigenstates of the problem are the odd $-n_{\text {, }}$ oscillator states. There are listed in portion (b) of Table I while the states with even z-parity are given in portion (c). As one can see the degeneracy patterns of both $n_{3}$-even and $n_{3}$-odd sets are the same and are exactly equal to the degeneracy of superdeformed oblate states in fig. 5 .

The analogy between the structure of superdeformed oblate states with $k_{\perp}=2$ and the harmonic oscillator with the barrier is by no means accidental. Indeed, the $N_{\text {shell }}$ quantum number (7) in the discussed case is equal to

$$
N_{\text {shell }}=n_{1}+n_{2}+2 n_{3}=n_{\perp}+n_{3}^{\prime}=N^{\prime},
$$

where $n_{3}^{\prime}=2 n_{3}$ is always even and $N^{\prime}$ is the principal quantum number of the oscillator with the barrier?. Ravenhall, Sharp an Pardee in ref. ${ }^{38}$ have shown that the resulting degeneracy pattern can be associated with two interleaving sets of irreps of $O(4)$. The generators of $O(4)$ are two vectors $J$ and $K$ generating rotations in the four-dimensional plane. By introducing two vector operators $J^{ \pm}=\frac{1}{2}(J \pm K)$ one can label the irreps of $O(4)$ using two quantum numbers $j^{+}$ and $j^{-}$. The corresponding dimensionality is $\left(2 j^{+}+1\right)\left(2 j^{-}+1\right)$. In the discussed case the angular momentum $J$ is nothing else but the farriliar quasispin $T$ genererating the $\mathrm{SU}(2)$ subgroup of $\mathrm{SU}(3)$. The components of the angular momentum $K$ have slightly more complicated form and they are given by. eq. (10) of ref. ${ }^{38}$.

\footnotetext{
This interesting analogy has been firat noticed by Cisneros and MeIntosh, ref. ${ }^{28}$, p. 891
} 
The eigenstates of superdeforned oblate RHO with $k_{\perp}=2$ form the basis for two irreps of $\mathrm{O}(4)$. Square irreps with $j^{+}=j^{-}=\frac{1}{4} N_{\text {shell }}$ arise when $N_{\text {shell }}$ is even whilst rectangular ones with $j^{+}=j^{-}+\frac{1}{2}=\frac{1}{4}\left(N_{\text {shell }}+1\right)$ correspond to even values of $N_{\text {shell }}$. The values of $j^{+}$and $j^{-}$can be expressed in terms of $\bar{N}$ and $\lambda_{\perp}$ as

$$
j^{+}=\frac{1}{2}\left(\bar{N}+\lambda_{1}\right), \quad j^{-}=\frac{1}{2} \bar{N}
$$

see fig. $5^{\prime \prime}$.

The degenerate shell of the RHO consists, in general, of states having different parities. Indeed, the generalized Bose operators (10) are parity-even for even values of $k_{i}$ and parity-odd for odd values of $k_{i}$ :

$$
\dot{\pi} A_{i}^{(\lambda)} \dot{\pi}^{-1}=(-1)^{k_{i}} A_{i}^{\{\lambda\}} \text {. }
$$

Consequently, the total parity of single-particle state $|\hat{n}\rangle$ can be written as

$$
\pi_{\dot{n}}=(-1)^{N}=(-1)^{\lambda_{1}+\lambda_{2}+\lambda_{3}}(-1)^{k_{1} \nu_{1}+k_{2} \nu_{2}+k_{3} \mu_{3}}=\pi_{\lambda} \pi_{\nu} .
$$

The above expression can be given a simple interpretation. The parity $\pi_{\lambda}$ is the intrinsic parity of the corresponding bosonic vacuum whilst $\pi_{\nu}$ represents the parity of an excited mode. In the case of superdeformed prolate shapes with even values of $k_{3}$, eq. (26) reduces to

$$
\pi_{n}=(-1)^{\lambda_{3}}(-1)^{n_{\perp}}=(-1)^{\lambda_{3}+\Lambda},
$$

where $\Lambda$ is the projection of the orbital angular momentum on the symmetry axis (z-axis).

The fact that the elementary modes of the RHO are nothing else but the $\mathrm{SU}(3)$ bosons has deep theoretical consequences since calculations at superdeformed shapes can be greatly simplified by introducing new symmetry-adapted operators constructed by means of new coordinates and momenta:

$$
X_{i}^{[\lambda\}}=\frac{1}{\sqrt{2}}\left(A_{i}^{\{\lambda]}+A_{i}^{[\lambda]+}\right), \quad P_{i}^{[\lambda]}=\frac{1}{i \sqrt{2}}\left(A_{i}^{[\lambda]}-A_{i}^{[\lambda]+}\right) .
$$

(Rasetti ${ }^{34}$ employed new coordinates $X_{i}^{(\lambda)}$ to construct the perturbation potential of the harmonic oscillator. States with different values of $k$ have been interpreted as " $k$-Boson clusters". In atomic physics the uncertainty in coordinates (28) is a direct measure of squeering, see e.g. ref. ${ }^{36}$.) Let us consider, for instance, the octupole mode, $r^{3} Y_{3 K}$. At the superdeformed shape with $k_{1}=1$

"It is well known that $O(4)$ is the dynamical symmetry of discrete states of Coulomb potential. On the other hand the unbound Coulomb atetes can be claenified using the infinite irreps of Lorens group. Interestingly enough the arial harmonic oncillator with the 2:1 axis ratio can be classified in vimilar way; on the oblate states the dynamical armmetry is $O(4)$, on the prolate side the eigenstates are bases of 5 wo irreps of Lorenr group, $T^{\left(\lambda_{3}\right)}$, wee ref. 30 , rol. 2 , wect. 20. 
Table I States of the spherical harmonic oscillator in representation (3). In (a), the etc. indicates permutations. The states in (b) are those remaining after inserting a wall in the $x y$-plare ( $n_{3}$-odd) whilst the states in (c) have even values of $n_{3}$. The degeneracy of the level is contained in the first column (cf. ref. ${ }^{38}$ ).

\begin{tabular}{|c|c|}
\hline Degeneracy & Cartesian representation of states \\
\hline & (a) Full three-dimensional oscillator \\
\hline 21 & 500 etc., 410 etc., 320 etc., 311 etc., 221 etc. \\
\hline 15 & 400 etc., 310 etc., 220 etc., 211 etc. \\
\hline 10 & 300 etc., 210 etc., 111 \\
\hline 6 & 200 etc., 110 etc. \\
\hline 3 & 100 etc. \\
\hline \multirow[t]{2}{*}{1} & 000 \\
\hline & (b) $n_{3}$-odd states \\
\hline 9 & $005,041,401,023,203,311,131,113,221$ \\
\hline 6 & $301,031,103,013,211,121$ \\
\hline 4 & $003,201,021,111$ \\
\hline 2 & 101,011 \\
\hline \multirow[t]{2}{*}{1} & 001 \\
\hline & (c) $n_{3}$-even states \\
\hline 12 & $500,050,410,140,104,014,320,230,302,032,212,122$ \\
\hline 9 & $400,040,004,310,130,220,022,202,112$ \\
\hline 6 & $300,030,210,120,102,012$ \\
\hline 4 & $200,020,002,110$ \\
\hline 2 & 100,010 \\
\hline 1 & 000 \\
\hline
\end{tabular}

and $k_{3}=2$, the $K=1$ and $K=3$ components conserve intrinsic parity $\pi_{\lambda}$ while the $K=0$ and $K=2$ interactions act only between states with opposite values of $\pi_{\lambda}$. Interestingly, at the superdeformed oblate shape with $k_{1}=2, k_{3}=1$, this scenario is reversed: the $K=0$ and $K=2$ modes conserve $\pi_{\lambda}$ (for more discussion see ref. ${ }^{10}$ ).

\section{MULTICLUSTER MODEL}

According to eq. (7) the energy difference between neighbouring oscillator shells, $\bar{w}_{\text {ahell }}$, decresses smoothly with deformation. This indicates that the overall 
magnitude of the shell effects is expected to be strongest at the spherical shape. Below are discussed some examples that indicate that evell at very strong elongations the appearing shell structure leads to an enhanced stability similar as for spherical shell gaps. Moreover, we will describe the deformed shell-stabilized systems in terms of "multiclusters" of spherical subsystems (clusiers), as dictated by the decomposition of the $\mathrm{RHO}$ representations into the isotropic ones, as described in the previous section. Of course, the term "cluster" should not be understood in the most direct sense of a spatial spherical cluster, since in medium mass and heavy nuclei the probability of clustering into large fragments is strongly inhibited by the Pauli principle. However, it turns out that the group-theory symmetries of these clusters induce some properties of superdeformed states as if the clustering occurred in the real space.

The main assumption of the "cluster" model is that every $\{\lambda\}$-family (an SU(3) oscillator) should correspond to an independent fragment. The number of fragments is then equal to the number of one-dimensional irreps of SU(3), i.e. it is equal to $k_{1} k_{2} k_{3}$. Of course, the clusters are not fully decoupled since the generalized Bose operators belonging to different values of $\{\lambda\}$ do not commute:

$$
\left[A_{i}^{\{\lambda\}}, A_{j}^{\left\{\lambda^{\prime}\right\}+}\right] \neq \delta_{\{\lambda\},\left\{\lambda^{\prime}\right\}}
$$

\subsection{LIGHT NUCLEI}

In light nuclei the spin-orbit interaction is relatively weak and, in addition, the diffuseness of the nuclear surface is comparable with the nuclear radius. Consequently, the simple harmonic oscillator model gives a fairly good approximation to the nuclear average potential.

A particular feature of light nuclei is that the rearrangement of a few particles may drastically change the shape. Among many well-deformed configurations in light nuclei, there are several good examples nicely illustating the scheme discussed in sect. 2 , namely:

- The superdeformed ground state of ${ }^{8} \mathrm{Be}$, which corresponds to two alpha particles side by side (fig. 6).

- The ground state of ${ }^{12} \mathrm{C}$, which resembles three $\alpha$-particles in a triangle, see fig. 6. This superdeformed oblate shspe $\left(k_{1}=k_{2}=2, k_{3}=1\right)$ can be associated with the superdeformed oblate gap at $N=3$. The calculated ground-atate quadrupole mowent, $-22 \mathrm{efm}^{2} 41,42$, agrees well with the experimental value, $-20.1 \mathrm{efm}^{2} 43$. At $I^{\pi}=4^{t}$ the hyperdeformed state $\left(k_{1}=k_{2}=1, k_{3}=3\right.$, three aligned alpha particles) becomes lowest in energy. The band-head of this very elongated structure is, most likely, the $0^{+}$resonant state at 10.3 $\mathrm{MeV}$ (cf. discussion in refs. ${ }^{44,45}$ ). 


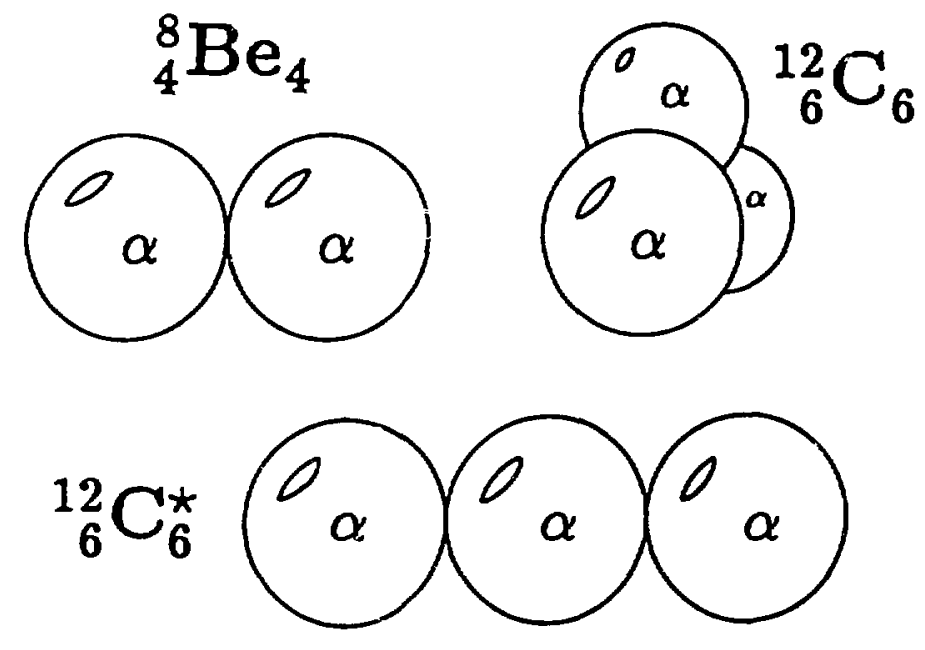

Fig. 6. Schematic representation of multi $\alpha$-cluster configurations in ${ }^{8} \mathrm{Be}$ and ${ }^{12} \mathrm{C}$.

- The ground-state superdeformed reflection asymmetric configuration of ${ }^{20} \mathrm{Ne}$, which can be well described as arising from an ${ }^{16} \mathrm{O}-{ }^{4} \mathrm{He}$ di-nucleus configuration. ${ }^{46-48}$ This results from the fact that the system composed of ten particles can exhibit shell effect when divided into two spherical "clusters" of two and eight particles, see fig. 3.

- The 4:1 state in ${ }^{16} \mathrm{O}$, which can be described in terms of the four aligned alpha particles. ${ }^{49-51}$

- The calculated low-lying reflection-asymmetric hyperdeformed minimum $\left(\varepsilon_{2}=1, \varepsilon_{3}=0.3\right)$ in ${ }^{24} \mathrm{Mg}{ }^{41}$ can be associated with the twelve-particle hyperdeformed gap. According to the simple oscillator scheme this configuration gives asymmetric ${ }^{16} \mathrm{O}+\alpha+\alpha$ (or ${ }^{16} \mathrm{O}+{ }^{8} \mathrm{Be}$ ) structures. The symmetric hyperdeformed $\alpha+{ }^{16} \mathrm{O}+\alpha$ states have also been considered ${ }^{52}$. Experimentally, resonances in the asymmetric fission ${ }^{24} \mathrm{Mg} \rightarrow{ }^{16} \mathrm{O}+{ }^{8} \mathrm{Be}$ (or ${ }^{20} \mathrm{Ne}+\alpha$ ) have been observed in the energy region of $14 \mathrm{MeV} \leqslant E_{x} \leqslant 28 \mathrm{MeV}$, cf. ref. 52 and refs. quoted therein.

- The ground state of ${ }^{28} \mathrm{Si}$, which can be associated with the superdeformed oblate gap occuring for fourteen particles, see fig. 5. The calculated groundstate quadrupole moment, $-66 \mathrm{efm}^{2} 41.42$, agrees well with the experimental value, $-64 \mathrm{efm}^{2} 53$.

- The weakly-deformed triaxial ground stete of ${ }^{32} \mathrm{~S}$ coexists with the lowlying superdeformed minimum $(\varepsilon=0.7) 41$. As demonstrated in 41 the superdeformed configuration, corresponding to the sixteen-particle superde- 
formed magic number (see fig. 3), is very stable with respect to reflectionasymmetric distortions. This state can be considered in terms of clustering into two ${ }^{16} \mathrm{O}$ substructures. ${ }^{52,54,55}$

\subsection{REFLECTION ASYMMETRY}

The role of spherical clusters in defining properties of superdeformed states becomes apparent when one considers the shell energy of the RHO. For magic numbers given by two unequal spherical clusters (case $A$ in fig. 3), i.e., $N$ or $Z$ equal to $28,60,110$ etc.), the shell energy should decrease with increasing reflection asymmetry. On the other hand, for the particle numbers 40,80, 140 (case $B)$, the nuclear shape is expected to be stable with respect to reflection asymmetric distortions. Calculations based on the realistic mean-field potentials confirm this general tendency, i.e. regions of particle numbers, which favour reflectionsymmetric or reflection-asymmetric shapes alternate. $37,45,56,57$

The shell energy of the Nilsson model is shown in fig. 7 (top) as a function of neutron number and octupole deformation, $\varepsilon_{3}$. Other deformation parameters are kept fixed at the values representative for superdeformed shapes, $\varepsilon_{2}=0.6, \varepsilon_{1}=0.09$. It is seen that the mass-asymmetry is strongly favoured at particle numbers around 28,64 and 114 whilst for particle numbers around 38,84 and 144 the minimum shell correction energy is found at $\varepsilon_{3}=0$, i.e. the microscopic calculations follow quite closely the harmonic oscillator pattern.

For hyperdeformed shapes, fig. 4, the harmonic oscillator model discussed in sect. 2 suggests that the strongest tendency for reflection asymmetry should be expected in case $A$, i.e. for the particle numbers $12,36,80,150$. Particle numbers that are most "rigid" with respect to octupole distortion (case $C$ ) are equal to $24,60,120$. The shell correction landscape computed within the Nilsson model is shown in fig. 7 (bottom) at deformation parameters, $\varepsilon_{2}=0.9, \varepsilon_{4}=0.203$, representative for hyperdeformed shapes. Again, like for the 2:1 shapes, simple predictions of the harmonic oscillator model are corroborated by the results of microscopic calculations, see also ref. 58 .

Nice examples of hyperdeformed structures can be found in the heavier actinide nuclei where a third minimum around the fission barrier has been calculated. 59.61 It corresponds to large elongations, $\beta_{2} \approx 0.9$, and pronounced reflectionasymmetry, $\beta_{3} \approx 0.2$. The third minimum is very localized in particle number and it is expected to be very shallow (the deepest minimum, about. $1.5 \mathrm{MeV}$, has been predicted ${ }^{61}$ for very neutron-rich nuclei around $Z=86, N=148$, i.e. exactly around the octupole-driving particle numbers 80 and 150). Experimentally, the third minimum shows up in the $(n, f)$ and $(d, p f)$ reactions as an alternating-parity microstructure of resonances. ${ }^{62,63}$ 

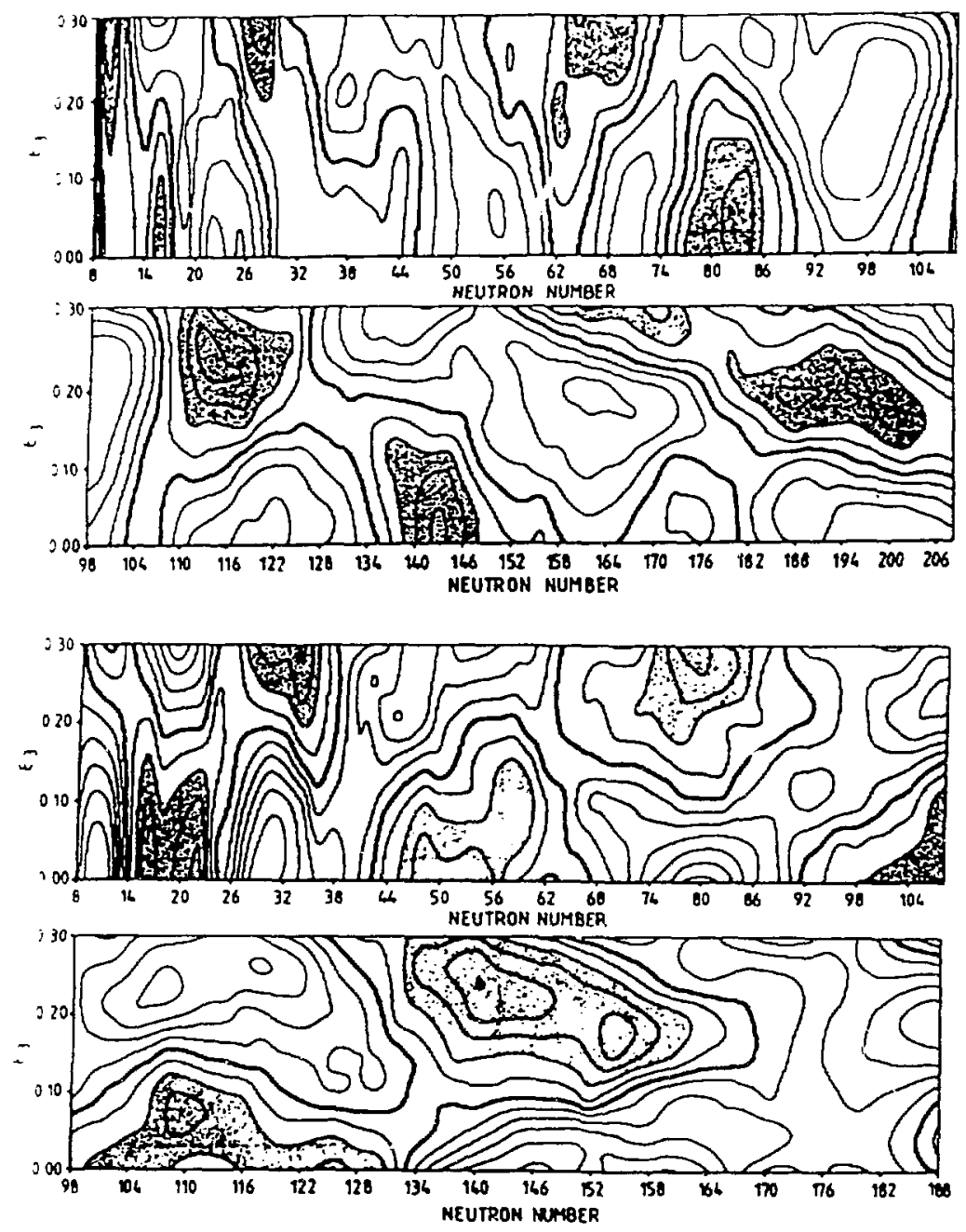

Fig. 7. The shell correction of the modified oscillator model as a function of neutron number and octupole deformation $\varepsilon_{3}$ for superdeformed (top, $\varepsilon_{2}=0.6$ ) and hyperdeformed (bottom, $\varepsilon_{2}=0.9$ ) shapes. (From ${ }^{37}$.)

\section{SUMMARY}

The atomic nucleus is a unique laboratory for studying quantum-mechanical symmetries. In addition to "normal" quantum numbers associated with basic nuclear symmetries, like angular momentum or parity, there are many other useful quantum numbers reflecting intrinsic symmetries of the nuclear mean field. For example, the single-particle states in nearly-spherical nuclei can be well classified by means of spherical quantum numbers $n_{r}, \ell, j$, but for deformed systems the asymptotic (or: pseudo-asymptotic) labelling $\left[N, n_{z}, \Lambda\right] \Omega$ is more appropri- 
ate. At high angular momenta the $\Omega$ quantum number is broken, but another selfconsistent symmetry is present, namely signature.

The symmetries of the RHO offer us a very convenient starting point to carry shell model calculations at superdeformed shapes. The new shell model basis can be very useful when diagonalizing realistic symmetry-dictated effective interactions, or can be helpful when discussing gross properties of elongated nuclei in the symmetry limit.

Another interesting observation is an apparent relation between the multiple irreps of $\mathrm{SU}(3)$ and the tendency (susceptibility) to cluster into spherical fragments. The simple relation between the cluster size, the number of clusters (equal to the number of irreps of $S U(3)$ ), and the overall equilibrium shape is supported by microscopic calculations. More details concerning this issue will be discussed in the forthcoming article $\mathbf{4 0}$.

Acknowledgements The Joint Institute for Heavy Ion Research has as member institutions the University of Tennessee, Vanderbilt University, and the Oak Ridge National Laboratory; it is supported by the members and by the Department of Energy through Contract Number DE-FG05-87ER40361 with the University of Tennessee. This project was supported in part by the Polish Ministry of National Education under Contract G-MEN-147/90.

\section{REFERENCES}

1. V.M. Strutinsky, Nucl. Phys. $\underline{\text { A95, }} 420$ (1967).

2. A. Bohr and B.R. Mottelson, Nuclear Structure, vol. 2 (W.A. Benjamin, New York, 1975).

3. B.T. Geilikma?, Proc. Int. Conf. on Nuclear Structure, Kingston, Canada, eds. D.A. Bromley and E.W. Vogt (Univ. of Toronto Press, Toronto, 1960), p. 874.

4. C.Y. Wong, Phys. Lett. $\underline{32 \mathrm{~B}}, 668$ (1970).

5. W. Názarewicz, R. Wyss and A. Johnson, Nucl. Phys. A503, 285 (1989).

6. I. Ragnarsson and S. Aberg, Phys. Lett. B180, 191 (1986).

7. T. Bengtsson, S. Áberg and I. Ragnarsson, Phys. Lett. 208B, 39 (1988).

8. K.T. Hecht and A. Adler, Nucl. Phys. A137, 129 (1969).

9. A. Arima, M. Harvey and K. Shimizu, Phys. Lett. 30B, 517 (1969).

10. R.D. Ratna-Raju, J.P. Draayer and K.T. Hecht, Nucl. Phys. $\underline{\text { A202 }}, 433$ (1973).

11. J.P. Draayer, Nucl. Phys. A520, 259c (1990). 
12. J.P. Draayer, these proceedings.

13. W. Nazarewicz, M.A. Riley and J.D. Garrett, Nucl. Phys. A512, 61 (1990).

14. J.P.Draayer, O. Castaños and S.C. Park, Contemporary Topics in Nuclear Structure Physics, eds. R.F. Casten, A. Frank, M. Moshinsky and S. Pittel (World Scientific, Singapore, 1988), p. 345.

15. J. Dudek, W. Nazarewicz, Z. Szymański and G.A. Leander, Phys. Rev. Lett. $\underline{59}, 1405$ (1987).

16. W. Nazarewicz, P.J. Twin, P. Fallon and J.D. Garrett, Phys. Rev. Lett. 64, 1654 (1990).

17. J.M. Jauch and E.L. Hill, Phys. Rev. $\underline{57}, 641$ (1940).

18. V.A. Dulock and H.V. McIntosh, Am. J. Phys. $\underline{33}, 109$ (1965).

19. Yu. N. Demkov, JETP $\underline{26}, 757$ (1954).

20. Yu. N. Demkov, JETP $\underline{36}, 63$ (1959).

21. G.A. Baker, Jr., Phys. Rev. $\underline{\text { C103, }} 1119$ (1956).

22. Yu. N. Demkov, Sov. Phys. JETP 17,1349 (1963).

23. G. Majella, Nuovo Cimento, $\underline{52 \mathrm{~A}}, 1004$ (1967).

24. I. Vendramin, Nuovo Cimento, $\underline{54 A}, 190$ (1968).

25. G. Maielle and G. Vilasi, Lett. Nuovo Cimento, 1, 57 (1969).

26. F. Duimio and G. Zambotti, Nuovo Cimento 48A, 1203 (1966).

27. R.A. Brandt and O.W. Greenberg, J. Math. Phys. 10, 1168 (1969).

28. L.A. Il'kaeva, Vestnik Leningrad. Univ. 22, 56 (1963) (in Russian).

29. A. Cisneros and H.V. McIntosh, J. Math. Phys. 11,870 (1970).

30. J.D. Louck, M. Moshinsky and K.B. Wolf, J. Math. Phys. 14, 692 (1973).

31. H.V. McIntosh, Group Theory and Its Applications, ed. by E.M. Loebl (Academic Press, 1971), vol, II, p.75.

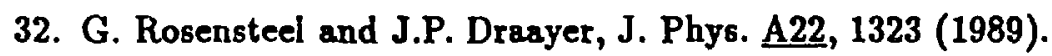

33. F. Iachello, these proceedings.

34. M. Rasetti, Int. J. Theor. Phys. 5, 377 (1972). 
35. J. Katriel, A.I. Solomon, G.D'Ariano and M. Rasetti, Phys. Rev. D34, 2322 (1986).

36. J. Katriel, M. Rasetti and A.I. Solomon, Phys. Rev. D35, 2601 (1987).

37. T. Bengtsson, M.E. Faber, G. Leander, P. Möller, M. Ploszajczak, I. Ragnarsson and S. Aberg, Phys. Scr. 24,200 (1981).

38. D.G. Ravenhall, R.T. Sharp and W.J. Pardee Phys. Rev. 164, 1950 (1967).

39. J.P. Elliott and P.G. Dawber, Symmetry in Physics (The Macmillan Press, London 1979).

40. W. Nazarewicz et al., to be published.

41. G.A. Leander and S.E. Larsson, Nucl. Phys. A239, 93 (1975).

42. Y. Abgrall, B. Morand and E. Caurier, Nuci. Phys. A172, 372 (1972).

43. A, Nakada et al., Phys. Rev. Lett. 27, 745 (1971).

44. I. Ragnarsson, S.G. Nilsson and R.K. Sheline, Phys. Reports $\underline{45}, 1$ (1978).

45. S. Åberg, Nucl. Phys. A520, 35c (1990).

46. F. Nemoto and H. Bando, Prog. Theor. Phys. $\underline{47}, 1210$ (1972).

47. S. Marcos, H. Flocard and P.-H. Heenen, Nucl. Phys. A410, 125 (1083).

48. D. Provoost, F. Grümmer, K. Goeke and P.-G. Reinhardt, Nucl. Phys. A431, 139 (1984).

49. Y. Fujiwara, H. Horiuchi, K. Ikeda, M. Kamimura, K. Sato, Y. Suzuki and E. Uegaki, Prog. Theor. Phys. Suppl. $\underline{68}, 29$ (1980).

50. S. Åberg, I. Ragnarsson, T. Bengtsson and R.K. Sheline, Nucl. Phys. A391, 327 (1982).

51. D. Auverlot, P. Bonche, H. Flocard and P.-H. Heenen, Phys. Lett. 149B, 6 (1984).

52. H. Flocard, P.H. Heenen, S.J. Krieger and M. Weiss, Prog. Theor. Phys. iㅡ, 1000 (1984).

53. Y. Hrikawa et al., Phys. Lett. $\underline{36 B}, 9$ (1971).

54. D. Baye and G. Reidemeister, Nucl. Phys. A258, 157 (1976).

55. D. Baye and P.H. Heenen, Nucl. Phys. $\underline{\text { A276, }} 354$ (1977). 
56. J. Dudek, T. Werner and Z. Szymański, Phys. Lett. 248, 225 (1990).

57. W. Satula, S. Ćwiok, W. Nazarewicz, R. Wyss and A. Johnson, Nucl. Phys. A (1991), in press.

58. J. Höller and S. Åberg, Z. Phys. A336, 363 (1990).

59. V.V. Pashkevich, Nucl. Phys. A169, 275 (1971).

60. P. Möller, Nucl. Phys. $\underline{\text { A192, }} 529$ (1972).

61. R. Bengtsson, I. Ragnarsson, S. Áberg, A. Gyurkovich, A. Sobiczewski and K. Pomorski, Nucl. Phys. A473, 77 (1987).

62. J. Blons, C. Mazur, D. Paya, M. Ribrag and H. Weigmann, Phys. Rev. Lett. $\underline{41}, 1282$ (1978).

63. B. Fabbro, J. Blons, A. Greiner, J.M. Hisleour, C. Mazur, Y. Patin, D. Paya and M. Ribrag, J. Physique Lett. $\underline{45}$, L-843 (1984).

\section{DISCLAIMER}

\footnotetext{
This report was prepared as an account of work sponsored by an agency of the United States Government. Nejther the United States Government nor any agency thereof, nor any of their bility for the accuracy, completeness, or usefulied, or assumes any legal liability or responsiprocess disclosed, or represents that its usefulness of any information, apparatus, product, or ence he in to any specific commercial use would not infringe privately owned rights. Refermanuf: tu er, or otherwise does not necessarily process, or service by trade name, trademark, mend: $\because$, or favoring by the United States Govstilute or imply its endorsement, recomand opinions of authors expressed herein United States Government or any agency thereof necessarily state or reflect those of the
} 